\title{
Face Detection Algorithm based on Skin Detection, Watershed Method and Gabor Filters
}

\author{
Abdellatif Hajraoui \\ « Information \& Telecommunication \\ Technologies Groupe» \\ Faculty of Science and Technology, University \\ Sultan Moulay Slimane, Beni Mellal 23000, \\ Morocco
}

\author{
Mohamed Sabri \\ « Information \& Telecommunication \\ Technologies Groupe» \\ Faculty of Science and Technology, University \\ Sultan Moulay Slimane, Beni Mellal 23000, \\ Morocco
}

\begin{abstract}
Automatic face detection has been intensively studied for human-related recognition systems. To build fully automated systems that analyze the information contained in face images, robust and efficient face detection algorithms are required. In this paper, a new face detection algorithm is proposed. This speedy and robust solution developed, on the one hand is based on the segmentation of the color image to skin regions using a new approach to detect the pixels of the skin and the water shed segmentation method. On the other hand, using Gabor filters, combined with a proposed model of face, skin regions are classified into two classes: face and non-face. The integration of these tools in our algorithm permits to develop a face detector with very reasonable and efficient performances. Experimental results show that the method mentioned in this paper can achieve high detection rates and low false positives. To evaluate the detection speed of proposed algorithm, a comparison with a recent known algorithm is made too.
\end{abstract}

\section{Keywords}

Human face detection, Skin detection, Watershed technique, Gabor filters.

\section{INTRODUCTION}

Face detection, a particular case of pattern recognition is a very important research topic in the field of image processing: It's a crucial step that comes before face recognition. It is pinpoints the location of every human face in a digital image or a video clip.

The quality of a face detection algorithm has a direct impact on the reliability and robustness of vision systems such as: video control based on biometric technology, humancomputer interaction, indexing images, video telephony, etc..

Despite the advancement of research in this area, robust face detection is an open-ended difficult problem at present because of the great variability of conditions of image acquisition, including the pose, orientation and size of the face in the image, the presence or absence of structural components, occlusions by other objects, facial expressions and illumination conditions.

To minimize or even eliminate these effects which degrade the performance of a face detection system, many techniques have been developed. Most of these approaches can be categorized into four main categories [1].

Among these, the class of methods based on invariant characteristics such as skin color, whose analysis turns out to be a good basic tool for multiple systems of face detection [2$6]$.

The algorithm proposed is in this category, because primarily uses a skin detector that covers the entire image. This choice is justified by the robustness of the color of the skin relative to geometric variations of the face model [7]. On the other hand its use as segmentation information improves the speed detection by reducing the search space of the face region in the image in comparison with other detection systems that run the image exhaustively, in all positions and for all possible sizes $[8,9]$. This superiority in terms of speed is demonstrated in the experiments section.

The common point between face detection systems that use skin color approach is the use of a skin detector. The construction thereof is made by a suitable color space and a definition of the distribution skin color model.

Several popular color spaces have been proposed in the literature. The RGB system is widely used because of its simplicity and the good results it produces [10-12]. Many researchers also recommend the normalized RGB system [13, 14]. The HSV color space is used frequently too in some works $[6,15]$. Finally, note that other papers [2, 5, 16-18] argue that the $\mathrm{YCbCr}$ color space provides good detection of skin pixels.

To model the distribution of color of the skin, several models have been proposed [19]. Including:

- Skin parametric model which adjusts the parameters of the distribution using some specific functions such as the Gaussian [20], a Gaussian mixture or an elliptical model $[2,18]$.

- Nonparametric skin model which estimates the distribution of skin colors in the training data without any assumption about the distribution, such as Bayesian model based on histograms [10].

- The skin fixed thresholds model that explicitly defines, following a number of rules, bounds of skin areas in the color space $[3,5,6,12-14]$.

\section{PROPOSED APPROACH}

The face detection algorithm proposed is shown in Figure 1. It contains two main modules:

(i) The segmentation of the color image to retrieve connected and significant regions representing objects of interest, consisting of skin pixels, allowing us to locate candidates face. 
(ii) The classification of skin regions into two classes: face or non-face.

The segmentation module (i) is done in four steps:

Step 1 Classify the pixels of the color image into two classes (skin / non-skin) using a skin detector, the result is a binary image.

Step 2 Segmenting the binary image, the result of the previous step, in connected and consistent regions using the well known segmentation technique: the line of the watershed.

Step 3 Elimination of non-significant regions.

Step 4 Location of significant skin areas.

The second module (ii) determines whether a face is present or not in each region of skin, localized or not, for submission to a cascade of Gabor filters and verification of correspondence with the face model suggested in this approach.

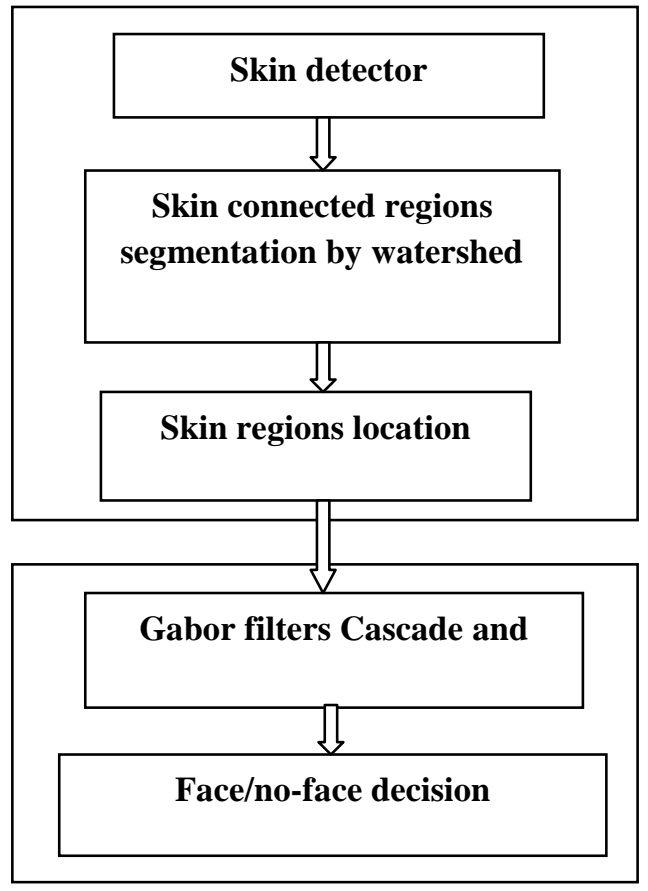

Fig 1: Block diagram of the proposed face detection approach

\section{SKIN DETECTOR}

The skin detector is a classifier which defines a binary space decision in the color space representation. Thus each pixel is classified as a skin pixel or not a skin pixel.

\subsection{Skin model}

The choice of a skin color model for a suitable color segmentation is always complex. In face detection, the detection of skin is just a preliminary step and should be fast while having the best performance in terms of true positive rate (TP: the probability that a pixel belongs to the skin class and is assigned to the skin class) and false positive rate (FP: the probability that a pixel does not belongs to the skin class and is assigned to the skin class). For this reason, the choice was focused on the skin model with fixed thresholds which produces a very fast classification due to the simplicity of the decision rules used to discriminate the pixels of skin from those of non-skin.
In addition to this quality, the skin model should bring up the facial characteristic features: the eyes and the mouth, because the model used for the discrimination of face and non-face is based on the characteristic features detection.

To achieve a model of skin color space suitable for detection system proposed, comparative tests were carried out first on four existing skin models using different color spaces.

To evaluate the performance (TP and FP) of these models, they were tested on a 200 color images: 100 images based Caltech_10K_WebFaces

(http://www.vision.caltech.edu/Image_Datasets/Caltech_10K _WebFaces/) and 100 captured images from the web.

\subsubsection{RGB model}

In RGB space, the model proposed by [12] is tested. This shows that the color level of the skin pixels must satisfy the following rules (R: red, G: green, B: blue):

$$
\begin{aligned}
& R>95 \text { and } G>40 \text { and } B>20 \\
& \max \{R, G, B\}-\min \{R, G, B\}>15 \\
& |R-G|>15 \\
& R>G \text { and } R>B
\end{aligned}
$$

\subsubsection{Normalized RGB model}

For the normalized RGB space, the model proposed by [13] is tested. The latter defines the skin model with the following bounds for the color levels:

$$
0.36 \leq \mathrm{r} \leq 0.465 \text { and } 0.28 \leq \mathrm{g} \leq 0.363
$$

With:

$$
r=\frac{R}{R+G+B} \quad \text { and } \quad g=\frac{G}{R+G+B}
$$

\subsubsection{HSV model (Hue Saturation Value)}

For the HSV space, the model proposed by [6] is tested. The latter defines the color level value ranges in the skin area as follows (H: The hue, $\mathrm{S}$ : the saturation, V: the brightness):

$0 \leq \mathrm{H} \leq 50,0.2 \leq \mathrm{S} \leq 0.68$ and $0.35 \leq \mathrm{V} \leq 1$

\subsubsection{YCbCr model}

In the same way, for the $\mathrm{YCbCr}$ space, the test is done with the proposed model in [5] which establishes the following rules for a skin color levels $(\mathrm{Cb}, \mathrm{Cr}$ : two chroma components from $\mathrm{R}, \mathrm{G}$ and $\mathrm{B}$ levels):

$$
97.5 \leq C b \leq 142.5 \text { and } 134 \leq C r \leq 176
$$

In Figure 2, some examples of the results of skin detection with the four previous models are showed.

\subsubsection{Discussion}

A synthesis of these results shows that the first model [12] using the RGB space ensures the best rate of TP but its weakness lies in the rate of FP. The latter is due to the false detection of the red or yellow pixels having colors close to skin one. However, this problem disappears in the normalized RGB space model [13].

\subsubsection{Proposed skin model}

Using both RGB and normalized RGB models, facial characteristic features can be detect. In order to combine the advantages of these two models, built the induction graph has been built (see figure 2) which can be rewritten as the following production rules: 
If (Pixel satisfies the condition C1)

Then

If (Pixel satisfies the condition C2)
Then skin class
Else

not skin class

Else not skin class

With:

\section{Condition C1}

$$
\begin{aligned}
& R>95 \text { and } G>40 \text { and } B>20 \\
& \max \{R, G, B\}-\min \{R, G, B\}>15 \\
& |R-G|>15 \\
& R>G \text { and } R>B
\end{aligned}
$$

\section{Condition C2}

$0.36 \leq \frac{\mathrm{R}}{\mathrm{R}+\mathrm{G}+\mathrm{B}} \leq 0.465$ et $0.28 \leq \frac{\mathrm{G}}{\mathrm{R}+\mathrm{G}+\mathrm{B}} \leq 0.363$

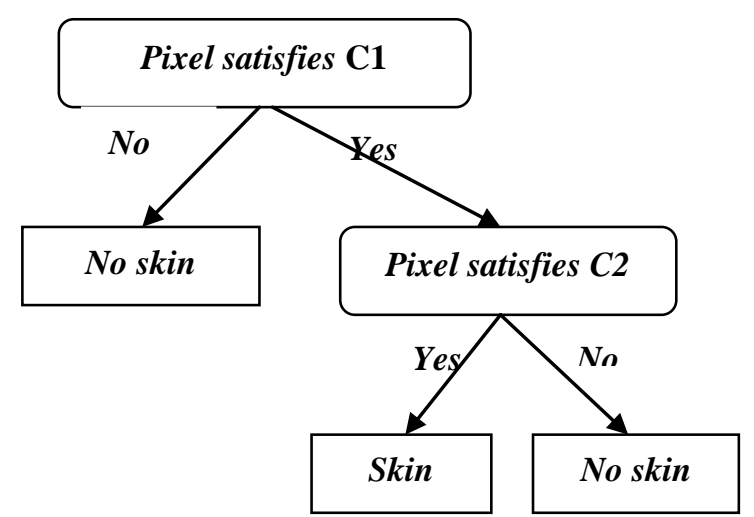

Fig 2: The inducing graph of the proposed skin model

This skin model allowed us to obtain very satisfying results in terms of TP and FP rates. Figure 3-f shows an example of detection using our algorithm.

\section{SEGMENTATION OF THE BINARY IMAGE INTO CONNECTED AND SIGNIFICANT SKIN REGIONS}

The previous step has allowed us to locate skin pixels. The recognition of skin areas must now proceed.

To this end, a segmentation is performed to extracts meaningful and coherent regions representing objects of interest and composed of skin pixels.

\subsection{The line watershed technique}

As a method of image segmentation, the line watershed technique is used, which finds its origin in mathematical morphology.

One finds in the literature two main classes implementing the LWS, from which derive several versions: one based on the use of geodesic distance functions and the other on an immersion recursive algorithm [21].

In this approach, the second class is opted because of its relevance and simplicity. Indeed, in this algorithm, an image can be seen as a relief by associating the gray level of each pixel at an altitude. It is then possible to define the line of the watershed as the ridge forming the boundary between two watersheds.

However, the algorithm derived from the LWS method must process a grayscale image while we have a binary image (skin, not skin)!

The Euclidean distance transform solves this problem by generating a grayscale image from a binary image.

Sometimes called distance function, it allows an estimation of the distance between a given pixel and the nearest pixel of the image. Many image processing algorithms rely on this transform to accelerate calculations such as morphological operators and skeleton detectors.
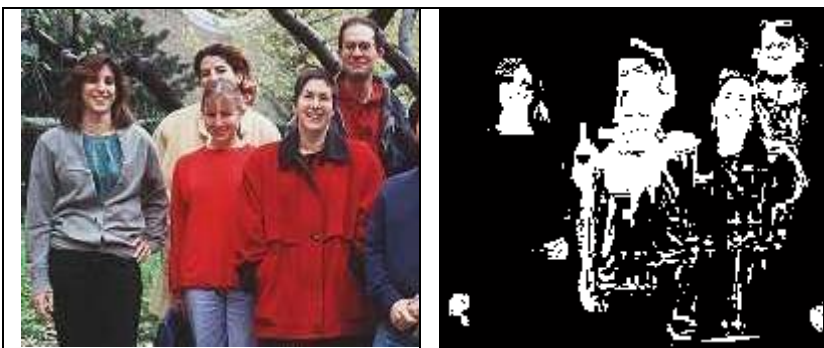

(a) (b)

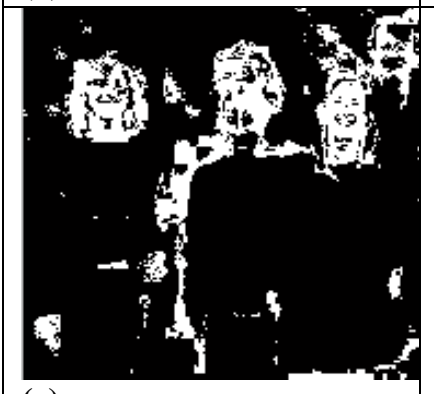

(c)
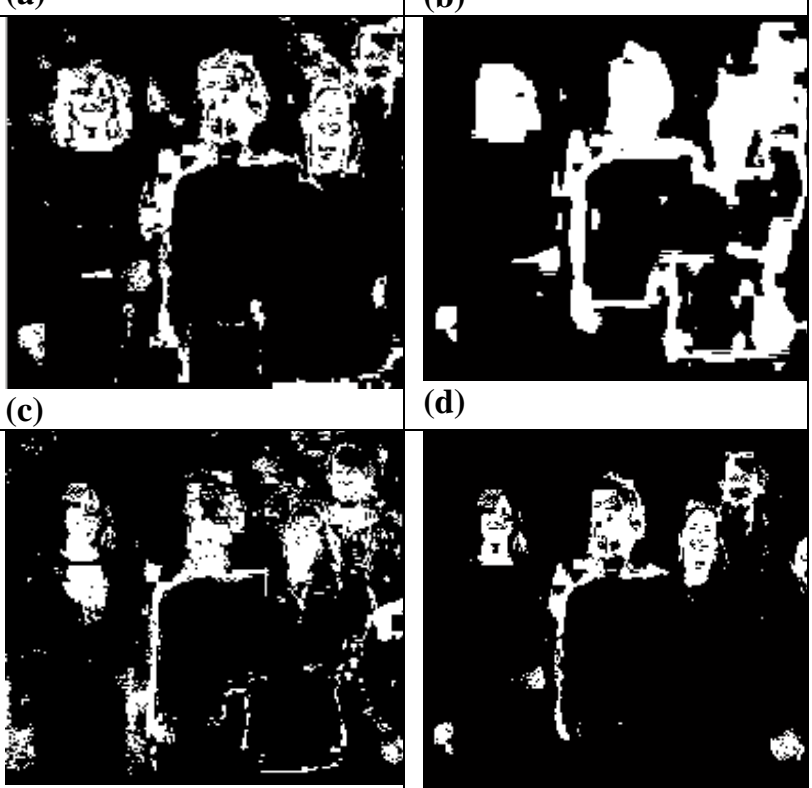

(d)

(e)

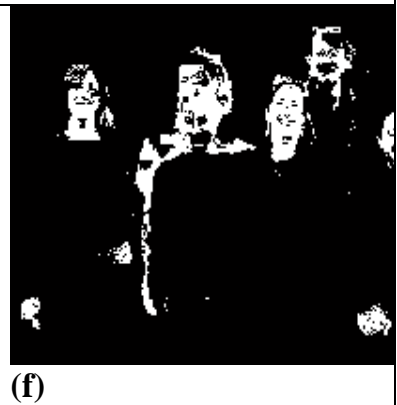

Fig 3: The skin detector: (a) original color image, (b) detecting based on RGB (c) detection based on standard RGB (d) detection based on $\mathrm{YCbCr}$ (e) detection based on HSV, (f) detection with proposed approach.

\subsection{The segmentation}

Once the segmentation by LWS, applied to the distance transformed image, is completed, all skin areas are masked to determine the most significant minimum size regions:

\section{Width $\geq 15$ and Height $\geq 15$}

Thus, the proposed algorithm does not detect a face smaller than $15 \times 15$ size, the other areas constitute potential faces. Figure 4 illustrates the segmentation steps mentioned previously. 


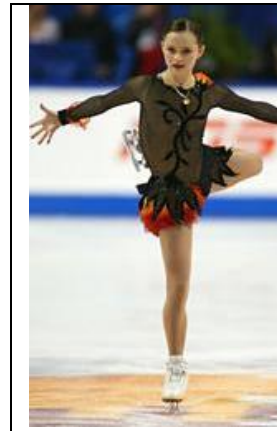

(a)

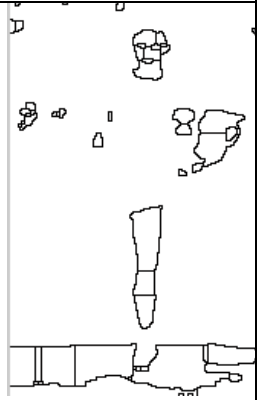

(d)

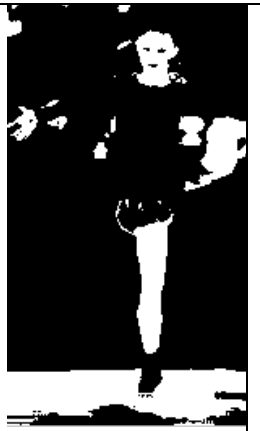

(b)

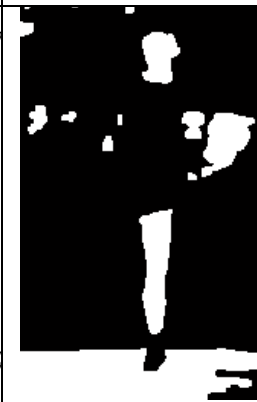

(e)

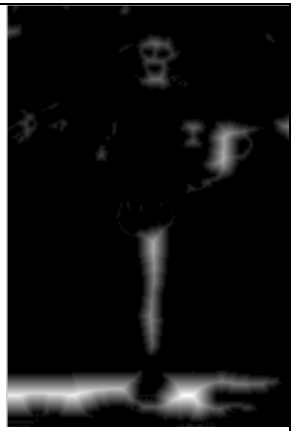

(c)

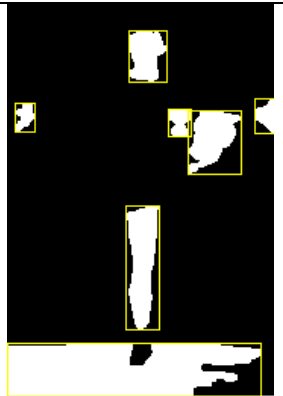

(f)
Fig 4: The results of the segmentation module steps: (a) original color image, (b) binary image of skin and nonskin pixels, (c) distance transformed image (d) segmented image by the LWS; (e) masked skin areas (f) image after removal of non-significant skin areas and location of significant skin regions.

\section{DETECTION AND LOCALIZATION OF THE FACE}

In this unit each of the localized areas of skin in the previous module is submitted to a cascade of four Gabor filters. At this point, the algorithm (see below) determines whether the treated skin area is a face or not as the result of similarity with suggested face model described below.

\subsection{Gabor Filters}

The 2-D Gabor filter (also called Gabor wavelets) is a Gaussian kernel modulated by a complex plane wave and parameterized with $(\mathrm{u}, \mathrm{v})$.

The expression of the filter in the image's spatial domain ( $\mathrm{x}$, y) is defined by:

$G_{u, v}(x, y)=\frac{f_{u}^{2}}{\pi k \rho} e^{-\left[\left(\frac{f_{u}^{2}}{k^{2}}\right) x^{\prime 2}+\left(\frac{f_{u}^{2}}{\rho^{2}}\right) y^{\prime 2}\right]} e^{j 2 \pi f_{u} x^{\prime}}$

With:

$x^{\prime}=x \cos \theta_{v}+y \sin \theta_{v}, \quad y^{\prime}=-x \sin \theta_{v}+y \cos \theta_{v}$

$$
\mathbf{f}_{\mathbf{u}}=\mathbf{f}_{\text {max }} / 2^{\left(\frac{u}{2}\right)} \text { and } \theta_{v}=v \pi / 8
$$

Where $f_{u}$ and $\theta_{v}$ denote the central frequency and the orientation of the complex plane wave. The parameters $\mathrm{k}$ and $\rho$ determine the ratio between the central frequency and the size of the Gaussian envelope along the $\mathrm{x}$ and $\mathrm{y}$ axes respectively.

The choice of different values for these parameters builds a bank of Gabor filters. Figure 5 shows the real parts and the amplitudes of $G_{u, v}(x, y)$ for 40 Gabor filters bank that have been built with the following parameters:

$k=\sqrt{2}, f_{\max }=0.25, u=0,1, \ldots, 4$ and $v=0,1, \ldots, 7$

The use of a Gabor filter bank is used to extract from the image relevant spatial and spectral information, thus providing a powerful tool for face recognition systems. The algorithms presented in this case in $[22,23]$ use the Gabor filters bank for extracting facial features in order to discriminate between faces of different people. The proposed algorithm, when with him, uses the filters bank to discriminate face objects of those non-face ones in the image.

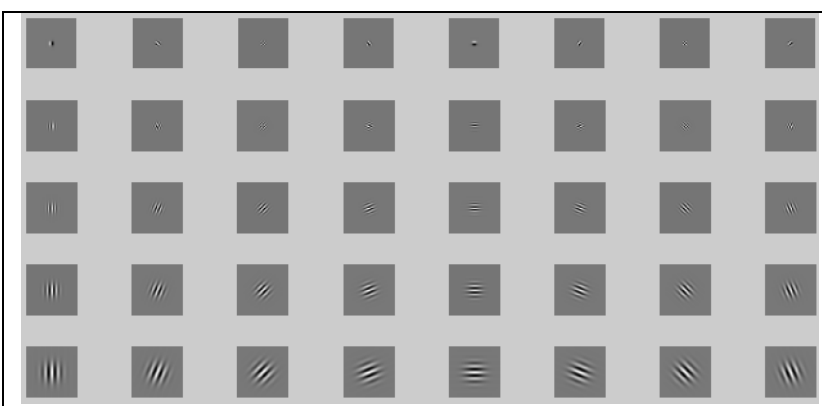

(a)

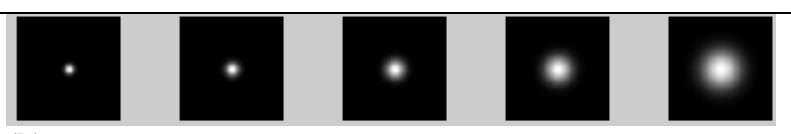

(b)

Fig 5: Representation of the 40 Gabor filters bank: (a) the real parts of the filters $(b)$ the amplitudes of the filters.

\subsection{Choice of Gabor filters Cascade}

In the second module of face detection system developed, the classification of skin regions localized in both face and a nonface class is done with a cascade of Gabor filters. The latter checks the presence and location of facial features, the eyes and the mouth, in a skin region.

To optimize the number of filters in the cascade, a series of filtering operations is conducted with the 40 Gabor filters bank, previously built, on 80 face images. This is illustrated in Figure 6

The binarisation of the Gabor filters outputs permits us to conclude that the best filter which detects the presence and location of facial features should have an angle of orientation $\boldsymbol{\theta v}$ satisfying the following relationship:

$$
\theta_{\mathrm{v}} \simeq \frac{\pi}{2}+\lambda
$$

Where $\lambda$ denotes the angle of inclination of the region of skin with respect to the vertical axis passing through the gravity center of said area (Figure 7). In addition, the amplitude of this filter depends on the size of the face and the quality of the output of the skin detector.

Examination of the results presented in Figures 7 and 8 affirm this conclusion. Using Figure 8, the three following actions must be made:

- Computing the inclination angle $\boldsymbol{\lambda}$ of the mask in the region of localized skin.

- Apply a rotation by an angle of $-\lambda$ to the skin region. 
- Subjecting the binary image, output of the skin detector, after its rotation to a cascade of four Gabor filters having the following respective amplitudes (parameterized by $\mathrm{u}$ ): $\mathbf{u}=\mathbf{0} ; \mathbf{u}=\mathbf{1} ; \mathbf{u}=\mathbf{2} ; \mathbf{u}=\mathbf{3}$ and having the same orientation $\mathbf{\theta v}=\mathbf{9 0}$ $\circ$.

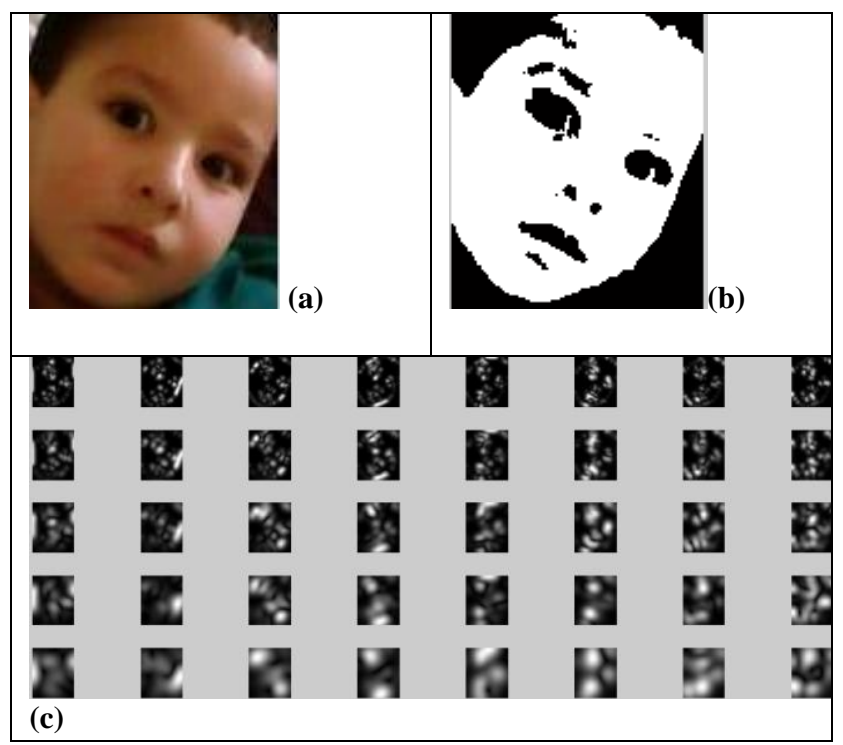

Fig 6: Result of filtering an image by the Gabor filters bank: (a) original color image, (b) binary image of skin and non-skin pixels, (c) the output images of $\mathbf{4 0}$ filters.

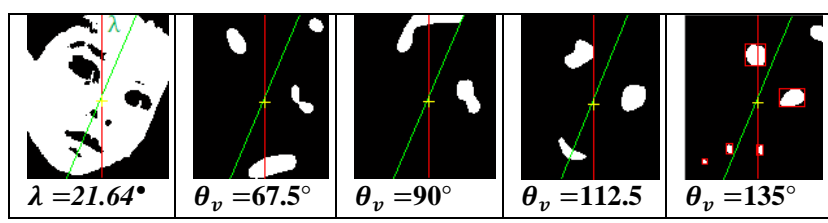

Fig 7: Filtering result of a face image, which has an inclination angle $\lambda=+21.64^{\circ}$ by filters having the same amplitude and different orientation values $\theta \mathrm{v}$.

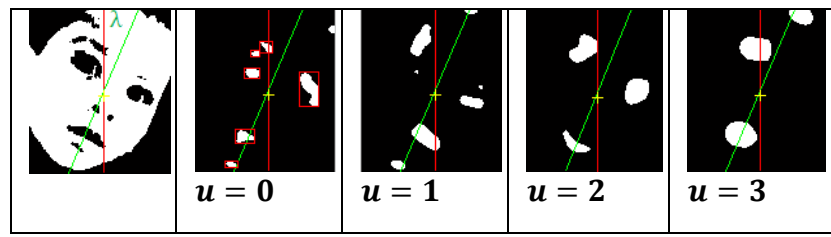

Fig 8: Result of filtering a face image with an angle of inclination $\lambda=+21.64^{\circ}$ by filters with the same value $\theta \mathrm{v}$ and different amplitude values (parameterized by $u$ ).

\subsection{The face model}

The final step of the proposed algorithm is the classification of the localized area of skin: face or non-face. To achieve this goal, we developed a face model which assigns the output of each Gabor filter of the cascade to the face class.

This model, shown in Figure 9, requires the simultaneous verification of the four following conditions:

Condition 1: The binary image of the filter output must contain at least three significant holes forming a triangle (not to mention the holes that are on the borders of the mask).

Condition 2: The vertical axis passing through the gravity center of the skin region mask must pass through the lowest hole among the three ones.
Condition 3: The two remaining holes must belong respectively to the two upper quadrants Q1 and Q2, formed by the two perpendicular axes passing through the center of gravity $\mathrm{G}$ of the triangle.

Condition 4: The two distances D1 and D2 of the latter two holes from the first one are to be close: $\mathbf{D 1} \simeq \mathbf{D 2}$.

\subsection{Decision flowchart «face or non face»}

The segmentation module allows segmenting the color picture into $\mathbf{N}$ connected and meaningful skin regions $\mathbf{R P}_{\mathbf{i}}(\mathrm{i}=1 \ldots \mathbf{N})$. Then, for each localized and extracted region of skin $\mathbf{R} \mathbf{P}_{\mathbf{i}}$, the treatment described by the flowchart in Figure 10 is performed to determine whether this region contains a face or not. If this is the case, the exact area of the face will be located.

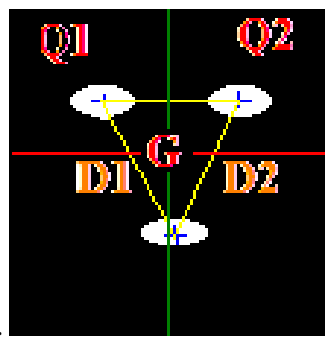

Fig 9: Face model.

\section{EXPERIMENTAL RESULTS}

The face detector developed is implemented under Matlab software version 7.11.0.584. The algorithm is tested with 400 color images, 200 images from the Database Caltech_10K_WebFaces and 200 images from the Web, in order to evaluate the following performances:

- The rate of true acceptance (RAR: RightAcceptance Rate), which expresses the percentage of real faces detected by the system.

- The false acceptance rate (FAR), which expresses the percentage of non-face regions detected by the system when they should be rejected.

- The average duration time of the face detection.

The results of RAR and FAR rates are shown in Table 1. To evaluate the detection speed of proposed algorithm a comparison with the Viola-Jones one [8] is presented in Table 2. Finally, a set of examples of face detection in color images are presented in Figure 11.

Table 1. RAR and FAR rates.

\begin{tabular}{|c|c|c|c|}
\hline $\begin{array}{c}\text { number of } \\
\text { faces per } \\
\text { image }\end{array}$ & $\begin{array}{c}\text { number of } \\
\text { images }\end{array}$ & RAR & FAR \\
\hline 1 & 123 & $99,18 \%$ & $0 \%$ \\
\hline 2 & 102 & $98,02 \%$ & $0,98 \%$ \\
\hline 3 & 85 & $96,86 \%$ & $1,56 \%$ \\
\hline More than 4 & 90 & $95,04 \%$ & $1,78 \%$ \\
\hline
\end{tabular}

Table 2. Evaluation of the speed detection.

\begin{tabular}{|c|c|c|}
\hline $\begin{array}{c}\text { number of faces } \\
\text { per image }\end{array}$ & Our algorithm & $\begin{array}{c}\text { Viola-Jones } \\
\text { algorithm }\end{array}$ \\
\hline 1 & $2.297 \mathrm{~s}$ & $5.048 \mathrm{~s}$ \\
\hline 2 & $2,342 \mathrm{~s}$ & $4.095 \mathrm{~s}$ \\
\hline 3 & $2.462 \mathrm{~s}$ & $4.703 \mathrm{~s}$ \\
\hline
\end{tabular}




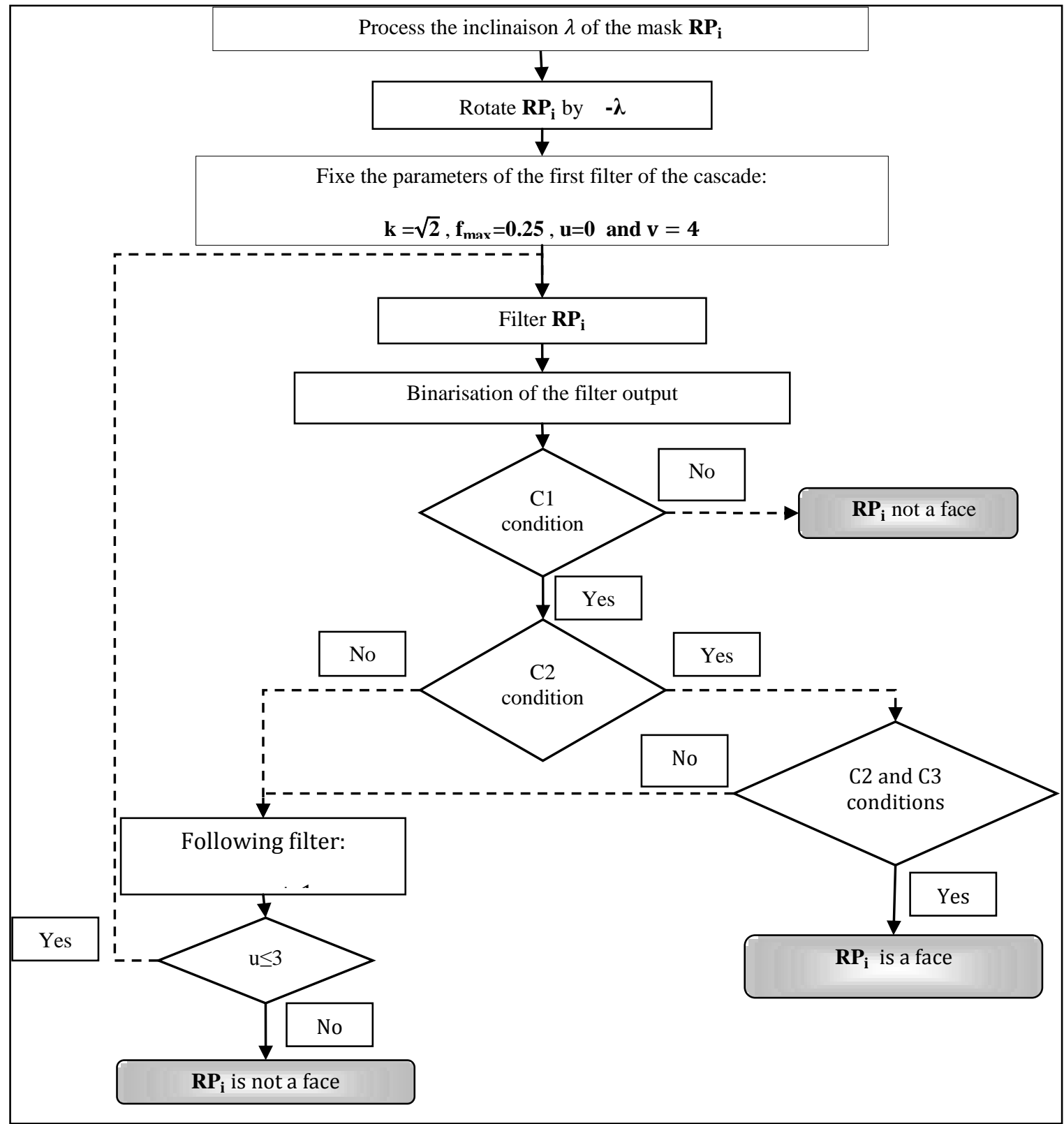

Fig 10: Decision flowcharts "face or not face"

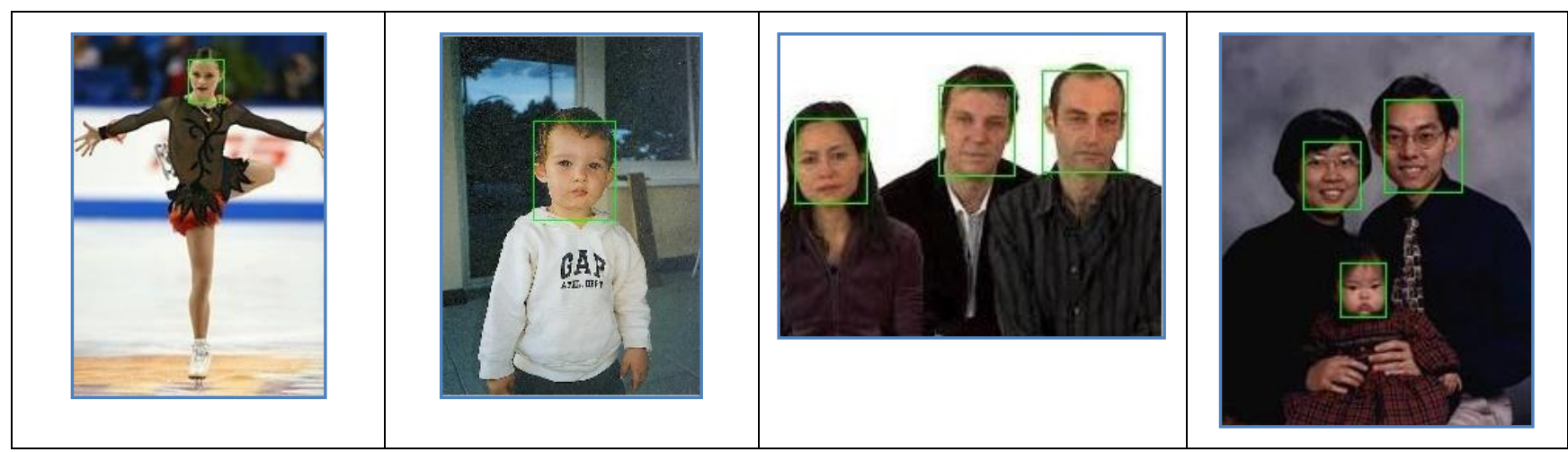

Fig 11: Examples of face detection with the proposed algorithm 


\section{CONCLUSION}

In this article, a robust solution is presented for the face detection problem, based partly on the segmentation of the color image into skin regions using a new approach to detect the pixels of the skin and the water shed segmentation method. Secondly, using Gabor filters combined with a face model, the skin regions is classified into two classes: face and non-face.

All these tools are well integrated into this system to develop a face detector with very reasonable performances. Indeed, the principle of segmentation reduces the search space of faces, which decreases the detection time duration. In addition, the use of Gabor filters to create a severe model of the face, to discriminate the skin regions, has prevented us to use a classification with a supervised learning, which conducts to confusion between the face class and non-face one. The strength of our system provides an excellent FAR rate.

The results obtained by the approach proposed are very encouraging, especially when the face is in profile in the image plane with any inclination angle.

To extend these results to images containing faces with different profile orientations, we are currently working on developing a new face model.

\section{REFERENCES}

[1] Yang M, Kriegman D, Ahuja N. Detecting Faces in Images: A Survey. IEEE Trans Pattern Anal \& Mach Intell 2002; 24: 34 -58.

[2] Rein-Lien H, Abdel-Mottaleb M, Jain A. Face detection in color images. IEEE Trans Pattern Anal \& Mach Intell 2002; 24: 696 -706.

[3] Kamarul H, Jie M, Rui X. An Innovative Face Detection based on Skin Color Segmentation. Int J Comput Appl 2012; 34: $6-10$.

[4] Yen-Hsiang C, Kai-Ti H, Shanq-Jang R, Statistical skin color detection method without color transformation for real-time surveillance systems. Eng Appl Artif Intell 2012; 25: 1331-1337.

[5] Hiremath P S, Ajit D. A Detection of multiple faces in an image using skin color information and lines of separability face model. Int J Pattern Recognit \& Artif Intell 2006; 20: 39-61.

[6] Wang Y, Yuan B. A novel approach for human face detection from color images under complex background. Pattern Recognit 2001; 34: 1983-1992.

[7] Vladimir V, Vassili S, Alla A. A Survey on Pixel-Based Skin Color Detection Technique. In: International Conference on Computer Graphics and Vision; 5-10 Sep 2003; Moscow, Russia.

[8] P Viola, M Jones. Robust real-time face detection. Int J Comput Vision 2004; 57: 137-154.

[9] Hong P, Yaping Z, Liangzheng X. Efficient and accurate face detection using heterogeneous feature descriptors and feature selection. Comput Vision Image Understanding 2013; 117: 12-28.
[10] J Brand, J Mason. A comparative assessment of three approaches to pixel-level human skin-detection. In: Proceedings of the 15th International Conference on Pattern Recognition; 3-7 Sep 2000; Barcelona, Spain: pp. 1056-1059.

[11] Michael J, James M. Statistical color models with application to skin detection. Int J Comput Vision 2002 46: 81-96.

[12] Kovac J, Peer P, Solina F. Human skin color clustering for face detection. In: IEEE Region 8 International Conference on Computer as a Tool; 22-24 Sept. 2003; Ljubljana, Slovenia: IEEE Region 8. pp. 144 -148.

[13] Jeonghee P, Jungwon S, Dongun A, Seongjong C. Detection of human faces using skin color and eyes. In: IEEE 2000 International Conference on Multimedia and Expo; 30 July - 02 Aug 2000; New York, NY, USA: IEEE. pp. 133-136.

[14] Aryanto S, Koichi Y. Skin Color Segmentation Using Coarse-to-Fine Region on Normalized RGB Chromaticity Diagram for Face Detection. IEICE Trans Inf \& Syst 2008; 91: 2493-2502.

[15] Sigal L, Sclaroff S, Athitsos V. Skin color-based video segmentation under time-varying illumination. IEEE Trans Pattern Anal \& Mach Intell 2004; 26: 862-877.

[16] Son Lam P, Bouzerdoum A, Chai D. A novel skin color model in ycbcr color space and its application to human face detection. In: IEEE 2002 International Conference on Image Processing; 22-25 sept 2002; Rochester, NY, USA: IEEE. pp. 289-292.

[17] Chai D, Bouzerdoum A. A bayesian approach to skin color classification in $\mathrm{YCbCr}$ color space. In: IEEE Region Ten Conference TENCON 2000; 24-27 Sep 2000; Kuala Lumpu, Malaysia: IEEE Region Ten. pp. 421-424.

[18] Jae Y Lee, Suk I Yoo. An elliptical boundary model for skin color detection. In: International Conference on Imaging Science Systems and Technology; 24-27 June 2002; Las Vegas, Nevada, USA.

[19] P Kakumanu, S Makrogiannis, N Bourbakis. A survey of skin-color modeling and detection methods. Pattern Recognit 2007; 40: 1106-1122.

[20] L Bergasa, M Mazo, A Gardel, M Sotelo, L Boquete. Unsupervised and adaptive Gaussian skin-color model. Image \& Vision Comput 2000; 18: 987-1003.

[21] L Vincent, P Soille. Watershed in digital spaces, an efficient algorithm based on immersion simulation. IEEE Trans Pattern Anal \& Mach Intell 1991; 13: 583 - 598.

[22] L Shen, L Bai, M Fairhurst. Gabor wavelets and general discriminant analysis for face identification and verification. Image \& Vision Comput 2007; 25: $553-$ 563.

[23] L Nanni, D Maio. Weighted sub-Gabor for face recognition. Pattern Recognit. Lett 2007; 28: 487-492. 